\title{
LA TEORÍA CRIÍTICA: UNA PERSPECTIVA PARA MEJORAR LA PRÁCTICA EDUCATIVA DE LOS MAESTROS*
}

Carolina Cecilia De la Hoz Campo ${ }^{1}$

* Este capítulo es resultado de la investigación doctoral realizada en el marco del Doctorado en Ciencias de la Educación de la Universidad de Caldas titulada "Las Prácticas educativas para la formación profesional, fundamentadas en el desarrollo de comunidades de aprendizaje y mediadas por la didáctica de proyectos".

1 Docente de planta de la Universidad Simón Bolívar. Instructora SENA. Docente Catedrática de la Universidad del Atlántico. Doctora en Ciencias de la Educación (RUDECOLOMBIA-Universidad de Caldas). Magíster en Educación (Universidad del Norte). Licenciada en Enseñanza de Tecnologías (Universidad del Atlántico). Tecnóloga Textil (Universidad Autónoma del Caribe). Miembro de los grupos de investigación "Educación, Ciencias Sociales y Humanas" y "Maestros y contextos - Diálogo y sociedad". Grupo GRACE, Universidad del Magdalena. cdelahoz30@unisimonbolivar.edu.co 


\section{RESUMEN}

En el marco de la investigación titulada "Las prácticas educativas para la formación profesional, fundamentadas en el desarrollo de comunidades de aprendizaje y mediadas por la didáctica de proyectos", se hizo un recorrido del concepto de práctica educativa según el constructivismo (histórico-cultural) y de acuerdo con la teoría crítica (histórico-social); se analizó la información, de lo cual se derivó una dialéctica complementaria que permitió fundamentar de una manera coherente y pertinente este estudio.

Palabras clave: Comunidades de aprendizaje, Comprensión, Socio-constructivismo, Teoría crítica, Transformación de las prácticas educativas.

\section{INTRODUCCIÓN}

Desde la estructura del título es viable entender su naturaleza y finalidad. Incluye las siguientes categorías: Las prácticas educativas porque se planteó un ejercicio de comprensión, de revisión y de su evaluación, para elaborar la propuesta metodológica orientada por la teoría de las comunidades de aprendizaje planteadas por Coll (1997) como un concepto más amplio de educación que requiere aceptar la necesidad de un proceso de reflexión y debate que conduzca al establecimiento de un nuevo contrato social por la educación. Esta necesidad implica una transformación de los procesos pedagógicos en la práctica educativa.

Se suscita entonces la reflexión sobre asuntos relevantes en el contexto educativo, que generan este estudio. Se desarrolló en tres dimensiones: epistémica, empírica y metodológica. 
La primera hizo referencia al conjunto de principios y conceptos de naturaleza teórica; la empírica se refirió a la red de prácticas específicas en el contexto social, y la metodológica se desarrolló en coherencia con el problema y los objetivos que orientaron el proceso investigativo.

Se propone responder a los retos educativos impuestos por las nuevas demandas sociales y laborales que emergen de los cambios socioeconómicos provocados por la globalización.

En este horizonte de necesidades y exigencias, se abordó el objeto de estudio de esta investigación en una doble dimensión: La dimensión epistémica a partir del referente teórico constructivista (socio-cultural) y socio-crítico (histórico-social) y en una dimensión empírica constituida por la red de prácticas de formación profesional en el SENA.

Se fundamenta epistémicamente la categoría de prácticas educativas como las acciones que dinamizan el proceso de enseñanza y de aprendizaje. Se forman en un espacio de interrelaciones entre los participantes y son abordadas según el constructivismo (histórico-cultural) y la teoría crítica (histórico-social) de Wulf (1997), para obtener una dialéctica complementaria que fundamentó la investigación.

Desde la Teoría Crítica, en cuanto se trató de un esfuerzo investigativo para sentar las bases conceptuales y promover prácticas educativas generadoras de autorreflexión de la praxis docente, y la transformación de las pautas y acciones presentes en la formación profesional hacia la configuración de comunidades de aprendizaje.

Las prácticas educativas desde el carácter histórico-social de 
la educación, generan el análisis de la dinámica entre las estructuras políticas y económicas y el cumplimiento de su papel sobre la educación. Una teoría crítica de la educación debe ayudar a los maestros en su práctica educativa. En consecuencia, surgen significados y prácticas heterogéneas que se debieron analizar y contrastar, para proponer la articulación de un planteamiento didáctico de conjunto y que sirvan para utilizar las comunidades de aprendizaje como un instrumento de cambio en la educación.

Es evidente que existe multiplicidad de teorías que fundamentan los procesos de enseñanza y de aprendizaje, posturas epistemológicas diversas, y distintas comprensiones. Sin embargo, algunas son obsoletas en la medida que no responden a los retos impuestos a la educación (consecuencia de la evolución de la sociedad, la influencia de las TIC y los efectos de la globalización...) para un satisfactorio desenvolvimiento social y laboral.

La investigación referenciada surgió del conocimiento, análisis y caracterización de las falencias de las prácticas educativas en la formación profesional, en particular en el ámbito institucional del SENA. Unas fragilidades y problemáticas expresadas en unas prácticas en las que predominan las acciones formativas tradicionalistas, y maneras intuitivas de concretar procesos de enseñanza y de aprendizaje.

La génesis de esta investigación se ubicó en la reflexión acerca de los procesos didácticos, y el desmejoramiento de los niveles de aprendizaje en torno a las prácticas educativas de la formación profesional, en oposición al convencimiento de la importancia de apropiarse de teorías y prácticas educativas efectivas. Las teorías de aprendizaje como la perspectiva de la teoría crítica son una respuesta al requerimiento que lleva a los docen- 
tes a concretar acciones fundamentadas epistemológicamente, revestidas de sentido y pertinencia con respecto a las necesidades sociales y laborales impuestas por la sociedad.

Ya en los años 60, Canonge y Ducel (1992) advertían sobre las dificultades con relación al impacto del avance tecnológico en la formación profesional. Ellos mencionaron una serie de obstáculos colectivos e individuales para el avance de las didácticas innovadoras. Entre los colectivos indica los paradigmas mentales, las actitudes, las tradiciones, los prejuicios y la falta de idoneidad. En el plano individual la resistencia al cambio, la ignorancia, la resignación, el sentimiento de impotencia, el conservadurismo técnico y la incapacidad. Estos fenómenos experimentados por los miembros de la comunidad educativa (directivos, instructores, aprendices, padres de familia) orientan la reflexión crítica que llevó a la pregunta investigativa.

Esta se cuestiona por los sentidos y significados de los docentes del nivel de formación profesional en relación con las prácticas educativas vigentes, para que al ser develados, se abra una nueva comprensión que fundamente y permita configurar una propuesta metodológica participativa, desde los argumentos teóricos de la perspectiva crítica y de las comunidades de aprendizaje para atribuir pertinencia en el desempeño profesoral e incidir en la transformación de los procesos, acciones y niveles de aprendizaje de la práctica educativa en la formación profesional.

En concordancia con lo planteado en el problema de investigación, los objetivos se estructuraron en un objetivo general que se propone coherente con la definición y delimitación de investigación. Los objetivos y preguntas de investigación deben 
ser congruentes entre sí e ir en la misma dirección. Los objetivos y preguntas de investigación establecen qué pretende la investigación (Hernández, Fernández y Baptista, 2010, p.365).

Como objetivo general se planteó el develar los sentidos y significados de los instructores del SENA en relación con las prácticas educativas vigentes para abrir una nueva comprensión que permita configurar una propuesta metodológica participativa, fundamentada en los argumentos teóricos de las comunidades de aprendizaje, e incidir en la transformación de los procesos, acciones y niveles de aprendizaje de la práctica educativa (formación por proyectos) en la formación profesional basada en competencias.

Considerando la descripción señalada en el problema de investigación, es necesario y urgente revisar los procedimientos que están adelantando los docentes del nivel de formación profesional, así como también la visión teórica que fundamentan estos procesos, que son los que direccionan las prácticas educativas, porque es indispensable responder a las necesidades educativas de una comunidad inmersa en un entorno con demandas laborales y sociales nuevas y exigentes, muy diferentes de las planteadas en las últimas décadas del siglo XX.

Ante esta situación se propone la configuración de comunidades de aprendizaje como innovación pedagógica que promueva acciones educativas acordes con las necesidades y características de la sociedad actual. En este sentido, Wenger (2001) considera que conocer requiere una participación activa en comunidades sociales. $Y$ le parece prometedor:

las maneras inventivas de hacer que los estudiantes participen en prácticas significativas, de proporcionar acceso a recursos que refuercen su participación, de ampliar sus horizontes para 
que se puedan situar en trayectorias de aprendizaje con las que se puedan identificar y de hacer que participen en acciones, discusiones y reflexiones que influyan en las comunidades que valoran. Valorar el trabajo de construcción de comunidades y procurar que los participantes tengan acceso a los recursos necesarios para aprender lo que necesitan aprender con el fin de actuar y tomar decisiones que involucren por completo su propio conocimiento. (p.27)

Estos fundamentos describen algunas razones de orden teórico que sustentan la conveniencia de la investigación. Esta mirada teórica proporciona una visión de la educación que pretende responder a los retos que plantea el nuevo escenario social, económico, político, cultural, laboral, apoyado por las tecnologías de información y la comunicación, TIC, y el paso de una economía industrial a una economía del conocimiento, lo cual requiere de nuevos escenarios y acciones acordes a los cambios mencionados.

Desde la delimitación contextual, la formación profesional es el nivel educativo en que analizarán las prácticas educativas y más específicamente en el SENA, institución formadora para el trabajo que ha ajustado su oferta a la demanda actual del sector productivo.

Sin embargo eso no es suficiente para ser competitivos, también hubo que contemplar las prácticas pedagógicas establecidas tradicionalmente y así buscar estrategias innovadoras para mejorar las formas de enseñanza y de aprendizaje, diseñando propuestas contemporáneas que incluyan la pedagogía y la didáctica. Asumir los retos que plantea la sociedad en la acción educadora, implica conocer los desarrollos y transformaciones de las prácticas educativas en los distintos períodos históricos. 
La evolución y desafíos educacionales exigen transformaciones en el nivel pedagógico, curricular y didáctico para alcanzar los objetivos de formación que apunten a formar jóvenes reflexivos, creativos y autónomos. Por consiguiente, las prácticas educativas deben ser consideradas -en su complejidad- en la interrelación de los docentes y estudiantes con el conocimiento.

Sin embargo, a pesar de todos los recursos y actualizaciones para mejorar la actividad de formación, existen fragilidades en la práctica pedagógica de los docentes porque hace falta un proceso de reflexión profundo sobre las prácticas educativas, que les permita pensarse en relación con los demás, lo cual ayuda a mirarse para poder transformarse y transformar. Es esta necesidad la que promueve la indagación epistemológica desde la teoría crítica.

En la medida en que la teoría crítica comprende que la estricta división del trabajo entre teoría y ciencia, por un lado, y práctica social, por el otro, son características de una situación social particular, y por tanto cambiables, el valor de las teorías no se puede determinar únicamente en el marco de la investigación e independientemente de la práctica social. (Wulf, 1997, p.157)

Por lo anteriormente planteado, es vital una propuesta pedagógica que sirva de ruta metodológica para mejorar los niveles de enseñanza y de aprendizaje de todos los miembros de la comunidad del nivel educativo de formación profesional.

\section{Prácticas educativas según la teoría crítica} (histórico-cultural)

La teoría crítica de la educación es el fundamento epistemológico adoptado, porque concibe el pensamiento social en el terreno de la educación. Independientemente de la fuente o el origen de la ciencia crítica de la educación hay elementos comunes a las diversas fuentes. 
Según Wulf (1997), se requiere la reflexión sobre los límites sociopolíticos de las ideas pedagógicas, por lo cual rechazó la autonomización idealista de lo pedagógico en relación con lo social y la perspectiva de las finalidades de las prácticas educativas con sus condiciones sociales concretas. La teoría se entiende como una elaboración a partir de la práctica educativa. Se tiene en cuenta la intervención tecnológica, las ideologías, los objetivos educativos y la "responsabilidad pedagógica", cuya evaluación se hace en relación con su práctica.

El referente principal es el sujeto en su devenir, de lo cual surge la pregunta clave de todo proceso educativo: ¿Cómo estructurar el campo pedagógico para ayudar al sujeto que se educa a desarrollar su conciencia y a construir su identidad histórica?

Según la concepción socio-crítica, los procesos de autorreflexión y autocrítica son permanentes. Esta es una teoría de la práctica educativa por y para la práctica que permite detectar lo que es posible en un contexto social determinado para mejorar la praxis educativa en sus rasgos característicos y permanentes. Esta teoría contempla, entre las diversas orientaciones que influyeron, y aún actualmente lo hacen, la ciencia de la educación; la teoría crítica (Horkheimer, 2000), la dialéctica de la ilustración (Horkheimer \& Adorno, 2007), la dialéctica negativa (Adorno, 1975), el hombre unidimensional (Marcuse, 1972) y la relación entre el conocimiento y el interés (Habermas, 1982). No se hará referencia a cada una de estas orientaciones, sin embargo, se señala el estímulo de los trabajos de Habermas (1988) a la teoría crítica. En sus trabajos, se hace implícito el pensamiento educativo de la escuela de Frankfurt² ${ }^{2}$ la cual afirma el carácter

2 La Escuela de Frankfurt surgió en el Instituto de Investigación Social de Frankfurt del Meno, como una consecuencia de los acontecimientos, que desde la década de los años veinte se iniciaban en Europa. Plantea la necesidad de desarrollar una reflexión global sobre los procesos que consolidan la sociedad burguesa-capitalista y el significado de la teoría ante tal consolidación. La Escuela devuelve a la Filosofía y a la Ciencia Social su carácter de análisis crítico, no solo en relación con la teoría, sino, también, con la praxis y la conjunción histórica de ambas. 
histórico y social de la educación, apoyándose en una teoría crítica de la sociedad, de la ciencia y del sujeto.

El aporte de Habermas es clave en las teorías del conocimiento, la comunicación, el lenguaje y en la de los roles, de la acción, de la socialización y la social. En su primera conferencia en 1965 en Frankfurt, posiciona una filosofía del saber y de la crítica social cuya finalidad es la autoreflexión de la ciencia. Allí establece un lazo estrecho entre teoría de la sociedad, filosofía crítica del saber y ciencia. El interés por la autonomía y la emancipación, es común a estos tres ámbitos en la medida en que estos no tienen en cuenta solo los procesos de formación individual, sino también los procesos de educación de la humanidad. Es solo con referencia al proceso de desarrollo de la especie que los procesos de formación y emancipación individuales se pueden evaluar convenientemente. Los procesos de formación individual dependen de una manera global, del estado de desarrollo histórico-social y de la situación de la "especie humana" (Wulf, 1997, p,138).

La idea anterior es central en la teoría crítica de la educación e implica construir un enlace entre razón y práctica, razón instrumental y razón crítica. Habermas (1982) explica que el interés rector del conocimiento puede tener varias justificaciones, un interés técnico (alcanzar una disposición lo más amplia posible sobre los objetos), un interés práctico (comprender las cuestiones

Para los filósofos y sociólogos que hacían parte de ésta, la crítica política era la pieza central de la teoría. La denominación de Teoría Crítica fue acuñada por Horkheimer y se extendió después como la definición más específica del sentido de la Escuela. Tanto Horkheimer como Adorno establecen el significado básico de "Teoría Crítica", esto es, el análisis crítico-dialéctico, histórico y negativo de lo existente en cuanto "es" y frente a lo que "debería ser", y desde el punto de vista de la Razón histórico-universal. Por tanto, para comprender el rumbo y la dinámica de la sociedad burguesa que se organiza económicamente a través del capitalismo, se hace indispensable la síntesis de las tres grandes concepciones críticas anteriores a la Escuela: Hegel-Marx-Freud aplicados dialécticamente en el examen de las direcciones de la relación entre racionalidad-irracionalidad y sus efectos sociales e históricos. 
primordiales de la vida), y un interés emancipatorio (eliminar la dominación humana).

El interés rector del conocimiento es explicado por Habermas así:

...no es un simple instrumento de adaptación de un organismo a un ambiente cambiante, ni el acto de un ser racional puro. El conocimiento es, sobre todo, una contemplación distante de los problemas de la vida. (Wulf, 1997, p.139)

Se plantean tres intereses cognitivos de forma coyuntural, como la expresión de una situación histórico-social en la que se hace una distinción entre el interés por la dominación del mundo, la comprensión humana y la emancipación.

Habermas se interesa por la función de la teoría científica contemporánea para la sociedad, pues la ciencia se ha convertido en una "fuerza productiva" instrumentalizada por el sistema dominante y sus intereses. La crítica de Habermas y de los científicos es científica y es ideológica. En él se enriquece la ciencia con la autorreflexión que incluye siempre, por anticipación, la crítica. Cuando "la idea de teoría de la sociedad esbozada en un objetivo práctico, se toma en serio, debe unir la pretensión de cientificidad a una estructura científica basada en la práctica" (Wulf, 1997, p.140).

En la transmisión de la teoría y la práctica, deben estar presentes teoremas críticos que resistan la crítica científica. Los teoremas deben ser utilizados y verificados en la reflexión de los grupos escogidos y la selección de estrategias apropiadas a la resolución de cuestiones tácticas.

En síntesis, la teoría crítica armoniza las afirmaciones verda- 
deras, verídicas y justificadas en un proceso de producción de conocimiento respecto a una teoría esbozada con sentido práctico. Y esto ocurre porque el objetivo no es la simple crítica, sino la organización de los procesos de explicitación y el desarrollo de estrategias aceptadas. La teoría crítica toma la práctica como campo de acción y esta es condición afín con la caracterización de la formación profesional que establece un período de práctica en el proceso formativo porque lo concibe como el espacio para hacer efectivas las teorías y los principios prácticos. En consecuencia, la relación teoría/crítica adquiere un nuevo matiz que deriva en un nuevo interés por la práctica educativa.

En el curso de la historia, la teoría crítica ha desarrollado una serie de conceptos de base que se encuentran estrechamente ligados los unos a los otros. Se trata de estructurar un encadenamiento del pensamiento para que los contextos de los conceptos respectivos resulten dentro de un contexto global. Entre estos está la relación de teoría y práctica que se encuentra en el centro de la teoría crítica y de las ciencias sociales críticas.

Desde la perspectiva de esta teoría, no basta con la producción de conocimiento, porque las teorías deben expresar un interés por el mejoramiento de las situaciones sociales, reflejando las condiciones de su producción y su utilización. En consecuencia, la teoría debe preguntarse por su fundamentación ideológica y sus posibilidades de impactar la práctica social. Las comunidades de aprendizaje son coherentes con la convicción de que la articulación entre la teoría y la práctica que han suscitado, responden a la necesidad del cambio educativo mencionado y que es indispensable concretar. La utilidad de las comunidades de aprendizaje reside en su posibilidad para cambiar las prácticas educativas y la organización habitual de las instituciones educa- 
tivas formales, como en su capacidad para movilizar recursos ajenos a estos centros educativos y ponerlos al servicio de la formación y la educación de las personas (Coll, 2001).

Sin embargo, las iniciativas de configuración de comunidades de aprendizaje como alternativa educativa, tienen orígenes disciplinares, teóricos y conceptuales distintos entre sí. Por esto, responden a preocupaciones educativas diferentes, implementación de distintas estrategias y conformación y desarrollos comunitarios diversos.

La teoría crítica parte de la evaluación del carácter histórico-social de la educación y de la interrelación entre el sistema educativo y la estructura de la sociedad. En este contexto, se hace un análisis sobre la influencia de los sistemas económicos y políticos en el sistema educativo. Surge así la pregunta por la función del sistema educativo en relación con la sociedad. "Una teoría crítica de la educación debe ayudar a los maestros en su práctica educativa" (Wulf, 1997, p.164).

La teoría crítica evidencia la verdadera situación social de la educación, mediante la crítica ideológica ${ }^{3}$. Esta debe atender múltiples objetivos: las teorías, los métodos, los planes de formación, los objetivos y los medios en el campo educativo, para ser analizados y develar los intereses sociales implícitos sobre ellos. En el centro de la reflexión está la denuncia de la opresión, de la injusticia social, del exceso de poder, de la reificación ${ }^{4}$ y de la autoalienación.

3 Según Wulf, este término significa un develamiento de las condiciones sociales de producción y de las racionalizaciones y los efectos de sus falsas interpretaciones, normas y teorías, que resultan de una comprensión ideológica de la situación social y de las posibilidades de intervenir sobre dicha situación.

4 Para Wulf, es la acción o efecto de convertir algo en cosa. En ocasiones, se utiliza también el término cosificación. En el contexto de la teoría crítica, es el resultado de la producción de bienes y el intercambio de bienes para mantener el orden social. 
La teoría crítica debe analizar sus causas sociales y sus expresiones institucionales para vislumbrar posibilidades de cambio y proponer objetivos constructivos para la educación.

Wulf (1997) concibe los objetivos de la teoría crítica, de modo que, además de la crítica a las estructuras perversas en el campo educativo, una teoría crítica de la educación está exigida igualmente a construir. No se puede limitar a la crítica ideológica, sino que debe desarrollar perspectivas de intervención que puedan servir como puntos de referencia para la crítica ideológica y la elaboración de una teoría de la educación. Porque más allá de la crítica a los procesos errados y a los conceptos insuficientes, implican una fundamentación que pueda servir de palanca para transformar la realidad.

El propósito de esta teoría es la crítica, y es también el mejoramiento de la práctica educativa. Este mejoramiento como objetivo de la teoría crítico-constructiva, ha conquistado su propio espacio de legitimación en el terreno de la investigación en educación. Con ella se pueden encontrar las pistas para una nueva práctica de la educación y así ayudar a los prácticos a mejorar su trabajo.

El objetivo central de la investigación, no es solamente un análisis que muestre la dependencia de los campos frente a las estructuras macro sociales, sino también el mejoramiento constructivo de las prácticas. Para alcanzar este objetivo, la teoría crítico-constructiva de la educación y la investigación a ella ligada se orientan hacia el nivel de acción de los prácticos. (Wulf, 1997, p.167)

En la presente investigación se ha descrito un problema en relación con las prácticas educativas, por lo cual se rastreó este concepto según las concepciones constructivista y socio-crítica. 
En esta, el valor de las teorías no se puede determinar solo en el marco de la investigación independientemente de la práctica educativa, pues tiene el deber de definir el valor de las teorías en la medida en que influye la práctica social. En ese sentido, se retoman esos argumentos explicativos frente al fenómeno objeto de estudio de este trabajo.

Considerando los aportes de Consuegra y otros (2015) se establece que:

la práctica sociocrítica se da a través de las didácticas, que propician los diálogos de saberes. Esta práctica construye el conocimiento a partir de la problematización que suscita un diálogo reflexivo como condición previa para abordarla, generando un ambiente de aprendizaje dialógico y democrático. (p.12)

Así mismo se han tenido en cuenta los aportes científicos de Alexander Ortiz (2011) con respecto a su análisis donde resalta que la conceptualización de qué es un modelo pedagógico facilitará identificar, valorar y elaborar modelos pedagógicos, con vista a obtener nuevos niveles de eficiencia educativa

Tal como se ha señalado anteriormente este estudio reflexiona las prácticas educativas del SENA y propone las comunidades de aprendizaje como innovación pedagógica para mejorar los niveles de aprendizaje en la formación profesional.

\section{METODOLOGÍA}

Desde el punto de vista metodológico, la investigación de referencia ha permitido proponer la categoría emergente de comunidades de aprendizaje, fundamentada desde la teoría crítica y la constructivista en torno a las prácticas educativas en la formación profesional. 
Dada la importancia de la doble función de la teoría en esta investigación, como insumo y producto, el propósito consistió en crear un entramado conceptual ligado a cada uno de los componentes de la investigación. La pretensión se centró en que el lector reconozca el devenir investigativo, con sus hallazgos, en coherencia con la fundamentación epistemológica que la orientó y los aportes teóricos que emergieron como producto de la investigación.

Obviamente esta intención teórica estuvo referenciada en contextos prácticos específicos que para el efecto se centraron en la formación profesional del SENA.

Metodológicamente, la investigación se estructuró teniendo en cuenta la naturaleza propia del objeto epistémico y empírico claramente delimitado (Sandoval, 1996).

En cuanto a los primeros, los objetos epistémicos, en esta investigación correspondieron a todo el constructo teórico-conceptual sobre los procesos de enseñanza y de aprendizaje y las prácticas educativas. Pensar las prácticas educativas ${ }^{5}$ planeadas y reflexionadas por los autores del proceso educativo desde el enfoque pedagógico en que se inscriben y enmarcadas en el contexto de la formación profesional ${ }^{6}$, requirió indagar rigurosamente su dimensión epistémica, la cual se explicará desde los referentes del enfoque constructivista ${ }^{7}$ socio-cultural, y el so-

5 Las prácticas educativas se definirán y analizarán ampliamente más adelante, sin embargo ahora se hace referencia a estas ya que son papel fundamental de la educación, en la construcción del conocimiento es un desafío su transformación e interrelación de los docentes y estudiantes con los conocimientos.

6 Se alude a la formación profesional porque es el contexto que enmarca la presente investigación, la cual se define como "el proceso educativo teórico-práctico de carácter integral, orientado.

7 Se recurre al principio constructivista para comprender y explicar mejor los procesos de desarrollo, de aprendizaje y los procesos educativos, así como para fundamentar propuestas de innovación en educación. 
cio-crítico ${ }^{8}$ histórico social para luego ser caracterizada desde su dimensión empírica.

La dimensión empírica de este objeto de estudio se hizo evidente en la manera en que los docentes implementan su acción de formación, y las actuaciones de los estudiantes en torno a las actividades formativas en los ambientes de aprendizaje. Si bien los procesos de influencia educativo se analizaron desde escenarios presenciales con didácticas tradicionales, actualmente se han venido realizando investigaciones sobre prácticas educativas en entornos de enseñanza y de aprendizaje no presenciales, ya sea a nivel virtual o híbridos, así como didácticas innovadoras, que plantean nuevos retos, tales como nuevas formas de interacción educativa, potenciadoras de los procesos de aprendizaje, además de ayudar a crear comunidades y redes de investigación en la educación.

El desarrollo del referente teórico de las categorías centrales de esta investigación y la aproximación al contexto empírico, sustenta este esfuerzo investigativo para responder a los retos de la educación actuales.

El proceso metodológico se llevó a cabo en coherencia con el problema y con los objetivos de la investigación; su diseño se estructuró en fases que se siguieron de manera progresiva y se llevaron a cabo de una forma dinámica cíclica (Bonilla, 2005), lo que promovió una permanente retroalimentación y ajuste al proceso investigativo. Esto se concretó en unos momentos donde se describieron las operaciones diversas que se adelantaron en cada una de las fases.

8 La concepción socio-crítica pone en evidencia las verdaderas condiciones sociales de la educación y debe visualizar la posibilidad de intervención y de cambio. 
Desde De Miguel (1989), se identifican tres fases constituidas por, la fase de decisión, aquí se identificaron los elementos que constituyen la formación profesional y se reflexionó sobre las prácticas educativas vigentes, implementadas por los docentes. Esto generó una concienciación de la situación en el contexto. Así surgió la decisión que determinó el problema de investigación, los objetivos, se delimitó el marco teórico y las actividades de la investigación. Siguió la fase de elaboración y ejecución del plan, que inició con un proceso de cuestionamiento de las prácticas educativas vigentes a la luz de los referentes teóricos que se estaban siguiendo, se hizo con la participación de los docentes involucrados en la investigación, y consistió en identificar los elementos constitutivos de sus prácticas educativas, confrontándolos con los postulados de aprendizaje emergentes de comunidades de aprendizaje en el marco de la teoría socio-constructivista y teoría crítica. Posteriormente se sistematizó la información obtenida y por último se hizo un plan de análisis para establecer la propuesta metodológica que resultó de esta investigación. Esta se diseñó de manera participativa e interactiva como una alternativa de actuación, a partir de la comprensión de los participantes sobre sus prácticas educativas en la formación profesional. Con base en la consideración de la secuencia anterior se finaliza con la fase de aplicación de los conocimientos; se llegó a analizar la propuesta metodológica diseñada desde las comunidades de aprendizaje, socializándola y aplicándola durante su elaboración. Esto fue el resultado de la interactividad que se generó en su elaboración con los participantes en la investigación hacia el colectivo de docente institucional.

\section{CONCLUSIONES}

La estructura de la investigación se cierra con algunas reflexiones obtenidas a partir de su fundamentación conceptual y 
metodológica. En esta dirección, es obligatoria la referencia a la pregunta que orientó la investigación, el cumplimiento de sus objetivos y los principales rasgos que describen las prácticas educativas que prevalecen en la formación profesional.

Un aporte importante es la propuesta metodológica de las comunidades de aprendizaje como práctica educativa innovadora para promover el mejoramiento de las acciones educativas en la formación profesional.

Como aporte académico se plantea la comprensión de las prácticas educativas vigentes en la formación profesional, resultado de su análisis que sustenta los principios básicos que marcan la pauta de prácticas fundamentadas en teorías emergentes.

El primer objetivo se centró en caracterizar los antecedentes y fundamentos teóricos que han marcado el desarrollo de las comunidades de aprendizaje como práctica educativa y los elementos de la formación profesional por competencias, mediada por proyectos formativos.

En relación con lo anterior, se realizó una rigurosa indagación de los fundamentos epistemológicos que sustentan las comunidades de aprendizaje, con el centro en la visión socio constructivista (Coll, 2001), la teoría crítica (Wulf, 1997) y los antecedentes, la conceptualización de la formación profesional y la didáctica de proyectos lo que implicó aplicar las técnicas de historias profesionales (Colás \& Buendía, 1994) con los investigadores del grupo CREA, para descubrir sus estructuras conceptuales y teorías que los animan a organizar los conocimientos elaborados en sus prácticas profesionales. En este horizonte, la participación de los integrantes del grupo CREA y de los instructores 
y coordinadores del SENA, mediante la técnica de historia de profesionales, permitió lograr el segundo objetivo.

El segundo objetivo fue referido a establecer los principales retos, necesidades, y escenarios de desarrollo de las comunidades de aprendizaje como práctica educativa, y que contribuyen a superar los agotamientos teóricos, pedagógicos y didácticos actuales en las prácticas educativas y articular aportes teóricos según las disciplinas que intervienen en el análisis, los enfoques y las metodologías que contribuyen a la consolidación de nuevas perspectivas y el desarrollo de propuestas educativas innovadoras. Así se ha contribuido a sentar las bases para la transición hacia un paradigma de comprensión de las prácticas formativas en el contexto de la formación profesional. Las bases se han expresado en los argumentos que se han incluido respecto a las categorías y principios epistemológicos que permiten abrir paso a la crítica de las prácticas educativas, lo que deriva en que se renueve un espacio discursivo del conocimiento como práctica social, que permite comprender la realidad y a partir de ello dotarla de nuevos sentidos.

El tercer objetivo específico, tiene que ver con la evaluación de las prácticas educativas actuales en el marco de la formación profesional y las necesidades de orientación teórica desde la perspectiva del desarrollo de comunidades de aprendizaje. Respecto a este objetivo, se logró una valoración crítica de los agotamientos teórico-prácticos como base para establecer los criterios conceptuales de nuevas prácticas educativas. Esto se hizo a partir del análisis de diversas tendencias teóricas que incursionan en la actual transición paradigmática del conocimiento, y de la lectura comprensiva de algunas prácticas educativas en 
la formación profesional con base en la revisión del Modelo Pedagógico Institucional -MPI-, el marco conceptual y pedagógico para la implementación de la formación por proyectos -FPP-en el SENA, y la sistematización de la experiencia de los instructores derivada de esta investigación, orientada a la renovación de las prácticas educativas.

Al inicio de la investigación, se hizo el diagnóstico, el reconocimiento, el análisis y la caracterización de la crisis de las prácticas educativas en el contexto de la formación profesional. El trabajo de campo realizado ha ratificado que dicha crisis se sigue expresando en prácticas educativas magistrales, con deficiencias en los procesos de inducción, planeación y ejecución de la didáctica de proyectos.

Retomando a Canonge y Ducel (1992), se encuentra que muchos académicos se resisten al cambio ya desde los años setenta, y advierten que el constante avance tecnológico crea obstáculos en la adopción de nuevos métodos educativos, entre los que están las mentalidades, las actitudes, la falta de idoneidad y las tradiciones que adoptan concepciones pedagógicas y prácticas obsoletas.

Uno de los mayores problemas de la teoría y la práctica en la formación profesional integral en ese actuar convencional que impide el desarrollo de las competencias de pensamiento establecidas para tal fin, para responder al reto que impone la sociedad actual, es la obsolescencia de las prácticas actuales por la insuficiencia conceptual y la escasa claridad instruccional respecto a los procesos formativos. A pesar de las transformaciones de los modelos pedagógicos, la incursión de las teorías de aprendizaje actuales y la posibilidad de las pedagogías activas, 
se sigue practicando una educación meramente operativa sin la participación activa, la reflexión y el desarrollo de habilidades de pensamiento.

Uno de los desarrollos derivados de esta investigación es el rastreo de las teorías de aprendizaje que fundamentan el desarrollo de comunidades de aprendizaje como una vía reflexiva, analítica y comprensiva que contribuya a la transformación de las prácticas educativas vigentes.

Mediante la investigación técnica y social elaborada en este estudio, se propone el mejoramiento de la calidad de las acciones y procesos pedagógicos con el soporte de una propuesta conceptual y metodológica de la formación profesional. Se trata de un ejercicio reconstructivo porque surgió de las experiencias educativas, de las indagaciones teóricas y de la formulación de operaciones para innovar las prácticas educativas.

Frente a los problemas de las prácticas educativas vigentes, la propuesta que se aporta en esta investigación es la metodología participativa, que le da sentido a unas prácticas innovadoras. Esta propuesta se elaboró recogiendo un conjunto de teorías de aprendizaje, centradas en teorías críticas, articulada con el análisis del contexto social para pensar nuevas posibilidades didácticas. De ese desarrollo conceptual, se pueden derivar transformaciones en las prácticas pedagógicas, lo cual inducirá a nuevas prácticas educativas, nuevos abordajes metodológicos, mejores maneras de aprender y la adopción de prácticas que promuevan la participación y la interacción de los participantes en la formación. 


\section{REFERENCIAS BIBLIOGRÁFICAS}

Adorno, T. (1975). Dialéctica Negativa. Madrid: Taurus.

Aubert, A., Flecha, A., García, C., Flecha, R. \& Racionero, S.

(2008). Aprendizaje dialógico en la sociedad de la información. Barcelona: Hipatia.

Bonilla, E. (2005). Más allá del dilema de los métodos: La Investigación en Ciencias Sociales. Bogotá: Norma.

Canonge, F., \& Ducel, R. (1992). La educación técnica. Barcelona: Paidós.

Colás, P. \& Buendía, L. (1994). Investigación Educativa. Sevilla: Alfar.

Coll, C. (1997). ¿Qué es el constructivismo? Buenos Aires: Magisterio del Río de la Plata.

Coll, C. (2001). Las Comunidades de Aprendizaje y el futuro de la educación: El punto de vista del fórum universal de las culturas. Simposio Internacional sobre Comunidades de Aprendizaje. Barcelona: Universidad de Barcelona.

Consuegra, J. y otros (2015). Proyecto Educativo Institucional de la Universidad Simón Bolívar. (Comp. Falla, S.). Barranquilla: Universidad Simón Bolívar.

De Miguel, M. (1989). La metodología de la investigación participante y desarrollo comunitario. Jornadas de educación permanente. Gijón: UNED.

Habermas, J. (1981). Teoría de la acción comunicativa, II. Madrid: Taurus.

Habermas, J. (1982). Conocimiento e interés. Madrid: Taurus.

Habermas, J. (1987). Teoría de la acción comunicativa. Madrid: Taurus.

Habermas, J. (1988). Teoría y praxis. Madrid: Tecnos.

Hernández, R., Fernández, C. \& Baptista, M. (2010). Metodología de la Investigación. México D. F.: McGraw-Hill.

Horkheimer, M. (2000). Teoría tradicional y teoría crítica. Barce- 
Iona: Paidós ICE/UAB.

Horkheimer, M. \& Adorno, T. (2007). Dialéctica de la Ilustración. Madrid: Akal.

Marcuse, H. (1972). El hombre unidimensional. Barcelona: Seix Barral.

Ortiz, A. (2011). Hacia una nueva clasificación de los modelos pedagógicos: El pensamiento configuracional como paradigma científico y educativo del siglo XXI. Revista Praxis, 7, 121-137.

Sandoval, C. (1996). Investigación Cualitativa. Bogotá D.C.: ICFES. Wells, G. (2001). Indagación dialógica. Hacia una teoría y una práctica socioculturales de la educación. Barcelona: Paidós.

Wenger, E. (2001). Comunidades de práctica. Aprendizaje, significado e identidad. Barcelona: Paidós.

Wulf, C. (1997). Introducción a la Ciencia de la Educación: entre teoría y práctica. Medellín: Universidad de Antioquia ASONEN. 